\title{
BMJ Global Health Initiative for Promoting Affordable and Quality Tuberculosis Tests (IPAQT): a market-shaping intervention in India
}

Harkesh Dabas, ${ }^{1}$ Sarang Deo (D) , ${ }^{2}$ Manisha Sabharwal, ${ }^{1}$ Arnab Pal, ${ }^{1}$ Sachin Salim, ${ }^{1}$ Lakshmi Nair, ${ }^{1}$ Kaartikeya Chauhan, ${ }^{1}$ Prateek Maheshwari, ${ }^{1}$ Aparna Parulkar, ${ }^{1}$ Ritu Singh, ${ }^{1}$ Manasi Chitalia, ${ }^{1}$ Rigveda Kadam, ${ }^{1}$ Manjot Kaur, ${ }^{1}$ Collins Oghor, ${ }^{3}$ Nirros Ponnudurai, ${ }^{3}$ Sameer Kumta, ${ }^{4}$ Peter Small, ${ }^{4}$ Puneet Dewan, ${ }^{4}$ Madhukar Pai ${ }^{3}{ }^{3}$

To cite: Dabas H, Deo S, Sabharwal M, et al. Initiative for Promoting Affordable and Quality Tuberculosis Tests (IPAQT): a marketshaping intervention in India. BMJ Global Health 2019;4:e001539. doi:10.1136/ bmjgh-2019-001539

Handling editor Seye Abimbola PD and MP contributed equally.

$\mathrm{PD}$ and MP are joint senior authors.

Received 28 February 2019 Revised 19 0ctober 2019 Accepted 25 October 2019

\section{Check for updates}

(C) Author(s) (or their employer(s)) 2019. Re-use permitted under CC BY. Published by BMJ.

${ }^{1}$ Clinton Health Access Initiative New Delhi, India

${ }^{2}$ Operations Management, Indian School of Business, Hyderabad, India

${ }^{3}$ Epidemiology and Biostats, McGill University, Montreal, Quebec, Canada

${ }^{4}$ Bill and Melinda Gates Foundation, New Delhi, India

Correspondence to Professor Sarang Deo; Sarang_Deo@isb.edu

\section{ABSTRACT}

A majority of patients with tuberculosis (TB) in India are diagnosed and treated in the private sector. Yet, most private providers do not use most recent WHO-endorsed microbiological tests such as liquid cultures, line probe assays and Xpert MTB/RIF due to a combination of factors such as lack of awareness, misaligned incentives and high prices that are unaffordable for patients. We designed a market-based approach to transform a highprice, low-volume market equilibrium into a low-price, high-volume equilibrium to improve the uptake of these tests. Toward this end, a non-profit consortium of private laboratories, called Initiative for Promoting Affordable and Quality Tuberculosis Tests (IPAQT) was formed in India in March 2013. It negotiated lower pricing on equipment and reagents with manufacturers, closer to that offered to the public sector. In return, IPAQT assured that this discount was passed on to patients, who typically paid for these tests out of their pockets, through an informally agreed on retail ceiling price. IPAQT also invested in demand generation activities that complemented the supply-side effort. IPAQT membership grew from 56 laboratories in 2013 to 211 in 2018. During this period, the initiative resulted in a 10-fold increase in the uptake of Xpert and a $30 \%-50 \%$ reduction in price. This initiative is planned to be expanded to other South Asian countries with similar TB epidemic and private market structure and dynamics. However, long-term sustainability of the initiative would require developing more cost-effective marketing activities and integration with broader private sector engagement agenda of the national TB programme.

\section{BACKGROUND}

The private sector is a major provider of healthcare in many countries with high burden of tuberculosis (TB) ${ }^{1}$ In these countries, patient pathways (including those for low-income segments) comprise visits to multiple providers leading to delayed diagnosis and suboptimal quality of care. ${ }^{2-4}$ Thus, large-scale engagement of private providers,

\section{Summary box}

A significant fraction of patients with tuberculosis (TB) in high burden countries are diagnosed and treated by private healthcare providers, whose uptake of WHO-endorsed TB tests is suboptimal due to low awareness and weak economic incentives driven by lack of affordability among their patients.

- A market-based initiative was developed in India wherein a consortium of private laboratories negotiated lower pricing from manufacturers and distributors and, in turn, agreed to provide lower ceiling price for patients.

- Over a period of 5 years, uptake of WHO-endorsed tests increased more than 10 -fold, along with a reduction in commercial price of the tests outside of the consortium.

- Financial sustainability of this initiative is likely to require integration with larger government efforts to engage with private providers, expansion to cover more tests and leveraging digital technology to increase awareness among private providers at scale.

including rapid scale-up of novel TB diagnostic tools, is critical for TB elimination by $2030^{15-7}$

In India, more than half of the estimated 2.8 million patients $(>25 \%$ of the global TB burden) are treated in a highly fragmented private sector with poor diagnostic and treatment practices. ${ }^{8-10}$ Most private providers diagnose $\mathrm{TB}$ based on a combination of chest X-ray, non-specific laboratory tests and empiric treatment instead of WHOrecommended sputum-based microbiological tests..$^{91-13}$ They also used antibody-based serological tests, despite the lack of clinical accuracy, until they were nationally banned in 2012. ${ }^{14-17}$ In contrast, WHO-endorsed tests such as Xpert MTB/RIF, line probe assays (LPAs), and liquid cultures experienced 

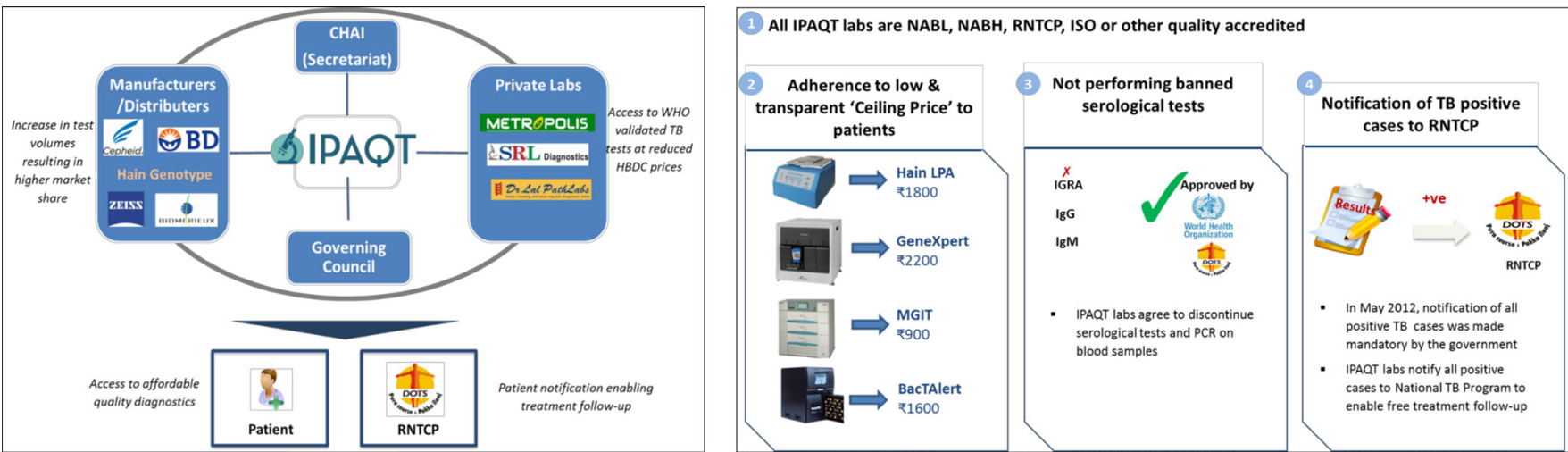

Figure 1 Governance structure and guiding principles of IPAQT. CHAl, Clinton Health Access Initiative; IPAQT, Initiative for Promoting Affordable and Quality Tuberculosis Tests; ISO, International Standards Organization; NABH, National Accreditation Board for Hospitals; NABL, National Accreditation Board for Testing and Calibration Laboratories; TB, tuberculosis; RNTCP, Revised National Tuberculosis Control Program.

limited uptake due to a combination of high-input pricing (compared with pricing for public and non-profit sectors), import duties, distributor and laboratory profits and physician incentives combined with limited willingness or ability of patients to pay for them out of pocket.

\section{OVERALL APPROACH}

We designed a market-based approach on the premise of existence of a large potential market for TB tests given the high TB burden in India and high use of private health services including laboratories. In particular, we attempted to increase the adoption of WHO-endorsed tests in the Indian private health sector by transforming a high-price, low-volume market equilibrium into a lowprice, high-volume equilibrium. The underlying theory of change was lower prices for high-quality tests in the private sector, combined with increased awareness of their benefits, will result in increased testing of patients with presumptive TB and sustained private sector uptake, provided that the earnings for all stakeholders in the diagnostic value chain (manufacturers, distributors, laboratories and providers) were protected and potentially enhanced by higher volumes.

Toward this end, a non-profit consortium of private laboratories called Initiative for Promoting Affordable and Quality TB Tests (IPAQT) was launched in March 2013 (figure 1). The governing council of the non-profit consortium comprised heads of select private laboratories, whereas the secretariat was managed by a not-forprofit entity, the Clinton Health Access Initiative. Funding for the secretariat was provided by international funding agencies, namely, the Bill \& Melinda Gates Foundation and Department for International Development (DFID). Technical and monitoring and evaluation support was provided by international research institutions, namely, McGill International TB Centre and Indian School of Business.

All IPAQT member laboratories agreed to abide by the following guiding principles: (1) have quality accreditation, (2) offer only high-quality (WHO-endorsed) TB tests, (3) notify patients with TB to the governmental health authorities and (4) comply with predetermined ceiling prices for tests. The secretariat undertook several supply-side and demand-side activities to facilitate implementation of these principles and increase adoption of quality TB tests, thereby creating a 'win-win-win' platform for all players in the value chain.

\section{Supply-side activities}

Before IPAQT, WHO-endorsed TB tests were supplied to the public sector in high-TB burden countries at prices that were significantly lower (the so-called 'High Burden Developing Country (HBDC) price') than those supplied to the private health sector providers. IPAQT negotiated with manufacturers (including BD, Cepheid and Hain LifeScience) to obtain concessional pricing (30\%$50 \%$ discount on existing commercial rates) on equipment and reagents for member laboratories, comparable with price offered to the public sector in high-TB burden low-income countries. In return, IPAQT provided assurance that this discount would be passed on to patients, who typically paid for these tests out of their pockets, and accordingly set the retail ceiling price. The manufacturers found this move to be incentive-compatible as the private market for these tests was small and the likelihood of private demand picking up at prevalent prices was low. Following the agreements with the manufacturers, margins of other channel partners (eg, distributors), including informal incentives between these stakeholders, were also reduced proportionally to support the lower retail price. Most distributors handled multiple diagnostic tests. Hence, they faced limited downside risk if the volume did not increase even after the reduction in the margin. The resulting retail prices for WHOendorsed TB tests were approximately $50 \%$ lower for Xpert MTB/RIF (US\$67-US\$33) and Hain Line Probe Assay (US\$58-US\$27) and approximately $15 \%$ lower for MGIT Liquid Culture (US\$18-US\$15).

\section{Demand-side activities}

IPAQT implemented several strategies to increase demand for quality $\mathrm{TB}$ tests among private providers. These were 
adapted from marketing and sales practices in the pharmaceutical industry and included continuing medical education (CME) seminars, focused group discussions (FGDs) and in-person visits by field sales force.

More than $120 \mathrm{CME}$ seminars were conducted in 35 cities between 2013 and 2016 by national and international experts to educate providers on WHO-endorsed diagnostic tests. Local IPAQT member laboratories were responsible for marketing these events among high potential providers in the city.

A field sales force with prior pharmaceutical sales experience was deployed between October 2014 and June 2016 in seven cities: Mumbai, Delhi, Patna, Coimbatore, Lucknow, Ahmedabad and Pune. It made routine visits to private providers and informed them on technical attributes of the tests, their availability at nearest IPAQT network laboratories and Standards of TB Care in India. In 2017, FGDs moderated by key opinion leaders were introduced to promote greater interaction and engagement among the participants.

Above routine activities were complemented by small experiments aimed at monitoring, learning and evaluation. Key examples include a randomised controlled trial to test the effectiveness of providing free samples (ie, vouchers for free Xpert tests) to providers in increasing their long-term adoption of tests and an external quality assurance study to assess the quality of Xpert MTB/RIF testing.

\section{NETWORK EXPANSION AND MARKET COVERAGE}

IPAQT membership grew from five laboratories in 2013 to 211 in 2018 (figure 2) with $>6000$ sample collection centres across 380 districts. These included seven national chains, 30 regional chains, 103 stand-alone laboratories and 69 hospital/medical college laboratories. Based on more than 30 responses to an online survey of member laboratories, we found 'access to newest technology at competitive prices' and 'social responsibility' as the most common reasons cited for joining IPAQT. However, IPAQT laboratories comprise only $\sim 20 \%$ of National Accreditation Board for Testing and Calibration Laboratories accredited private (national-chain, regional-chain and stand-alone) laboratories conducting microbiological testing laboratories and $20 \%$ of National Accreditation Board for Hospitals accredited private hospitals in tier II and III cities (cities with relatively lower population and lower cost of living). (This classification is based on the house rent allowance provided by the central.)

\section{TRENDS IN TEST VOLUMES}

IPAQT laboratories conducted around 620000 Xpert MTB/RIF, 47000 LPAs and 25000 BacTAlert Liquid Culture tests from 2013 to 2018. For all WHO-endorsed tests combined, testing volume increased from 28000 tests in 2013 to 275000 tests in 2018 (figure 2). Overall, national chain laboratories contributed $45 \%$ of IPAQT GeneXpert and LPA volumes. Three national chains, one regional chain and one hospital contributed $\sim 60 \%$ of the total volume. Xpert MTB/RIF contributed $>80 \%$ of test volume due to its ease of use, quick turn-around time, drug resistance markers and relatively lower technical requirements. Based on data obtained from Cepheid, we estimated that IPAQT contributed more than $80 \%$ of total Xpert volume in the private sector in India (figure 3). It is worth noting that this contribution has reduced slightly from $89 \%$ in 2013 to $81 \%$ in 2018 owing

\section{Growth in scale of the IPAQT network}

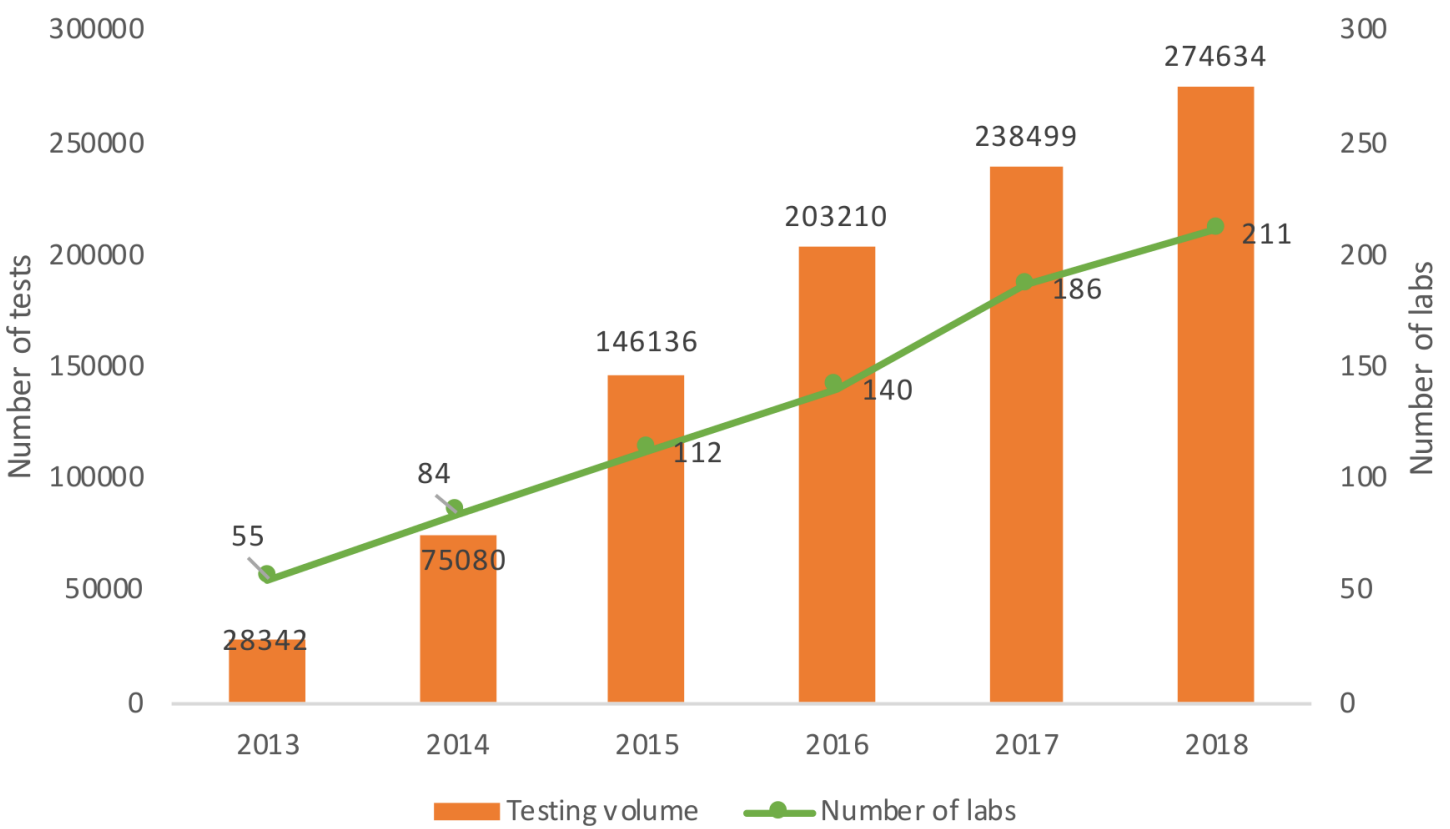

Figure 2 Growth in testing volume and number of laboratories in the IPAQT network. IPAQT, Initiative for Promoting Affordable and Quality Tuberculosis Tests. 


\section{Xpert Testing Volume (IPAQT and non-IPAQT)}

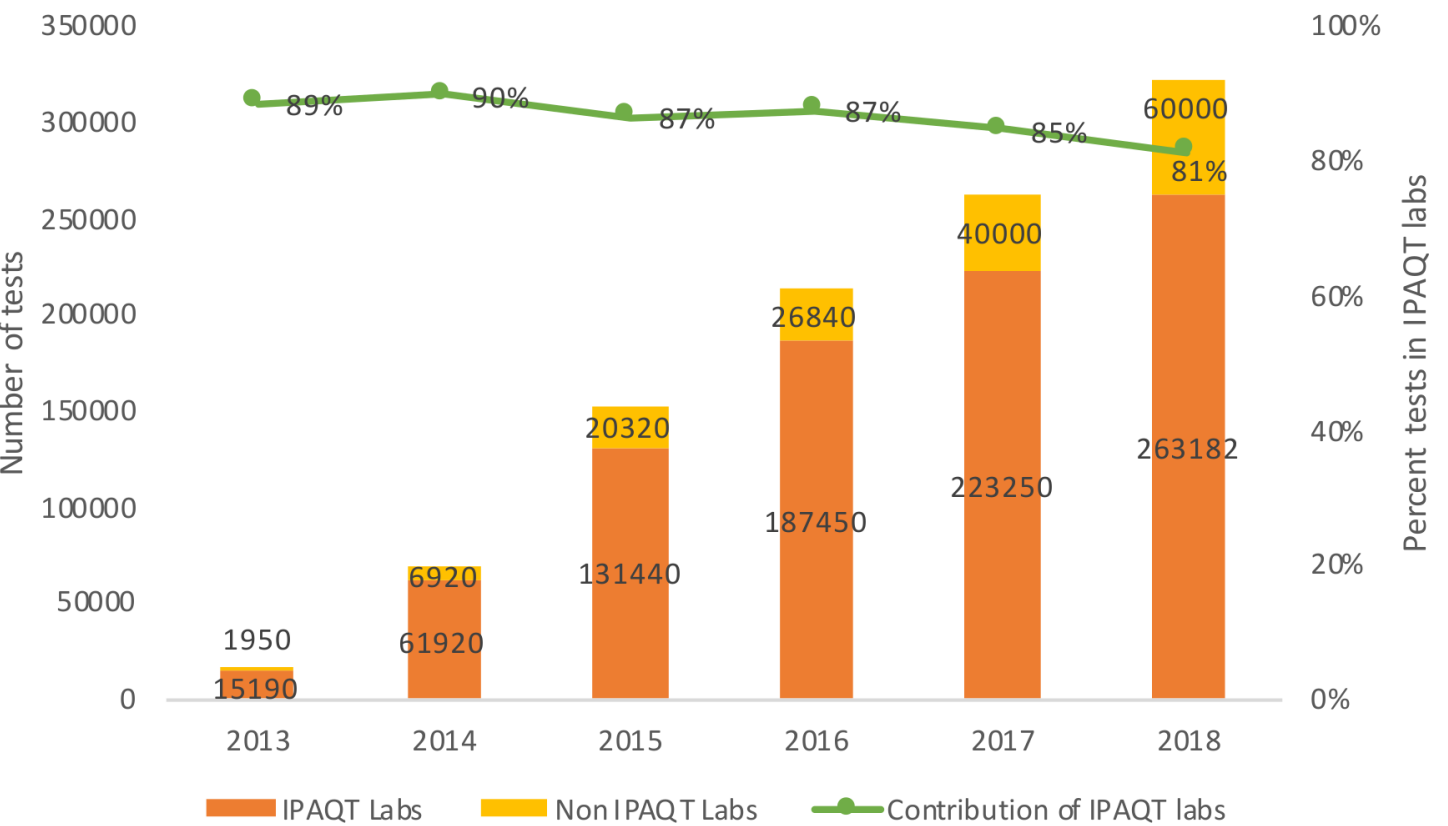

Figure 3 Contribution of IPAQT to Xpert MTB/RIF testing volume in India's private sector. IPAQT, Initiative for Promoting Affordable and Quality Tuberculosis Tests.

to rapid growth in Xpert testing volume in non-IPAQT laboratories. Moreover, compared with $>400000$ Xpert tests conducted by IPAQT laboratories in India during 2014-2016, sales in eight other high-burden countries were $\sim 156000$ tests (table 1 ).

\section{TRENDS IN TEST PRICES}

Based on data compiled from non-IPAQT laboratories, we found that IPAQT price of Xpert MTB/RIF (\$33.8) was consistently lower than non-IPAQT private market price (\$46.7) over the intervention period (figure 4). Interestingly, non-IPAQT price also experienced a substantial reduction from 2013 to 2017. This suggests that the lower pricing offered by IPAQT laboratories, which are dominant players in the market, may have created a downward

\begin{tabular}{|c|c|c|c|c|}
\hline Country & 2014 & 2015 & 2016 & $\begin{array}{l}2017 \text { (until } \\
\text { October) }\end{array}$ \\
\hline Myanmar & 1910 & 150 & 280 & 20 \\
\hline Indonesia & 200 & 50 & - & 50 \\
\hline Malaysia & 950 & 2910 & 3200 & 3727 \\
\hline Philippines & 2760 & 8820 & 14348 & 7860 \\
\hline Singapore & 1250 & 8178 & 12547 & 10000 \\
\hline South Korea & 25288 & 32971 & 39441 & 40099 \\
\hline Vietnam & 40 & 80 & 150 & 180 \\
\hline $\begin{array}{l}\text { Papua New } \\
\text { Guinea }\end{array}$ & - & 200 & 400 & 1400 \\
\hline Grand total & 32398 & 53359 & 70366 & 63336 \\
\hline
\end{tabular}

pressure on these commercial prices. These prices were also lowest among seven countries with a comparably sized private healthcare sector. ${ }^{7}$ Furthermore, the average price in those countries increased from $\$ 68.73$ in 2015 to $\$ 84.53$ in 2017 compared with the downward trend observed in India. We could not conduct a similar analysis for other tests as we did not have access to commercial pricing data for those tests from their manufacturers.

\section{LESSONS LEARNED}

Our results demonstrate the effectiveness and point to certain limitations of a market-based approach, in particular, the 'low-margin high-volume' strategy, to improve access to affordable and high-quality diagnostic tests among large and fragmented private sectors.

IPAQT was designed to be systematically different from previous interventions aimed at reducing prices of global health commodities, most notably the Global Drug Facility (GDF), due to key contextual factors. First, IPAQT was developed and implemented at a national level and hence had a much smaller scale compared with GDF, which is a multinational initiative. Second, on the supply side, a large part of price reduction was achieved by GDF through competitive tendering by suppliers. This was not possible in the IPAQT model due to the proprietary nature of each test. Instead, price reduction was achieved by the prospect of larger volumes, as well as lowering the cost for patients by guaranteeing that laboratories pass the benefits to the patients. Third, GDF's demand side partners were national TB programmes, which made uptake of the drugs and tests easier as the cost of these tools was not borne by patients. In contrast, IPAQT was 


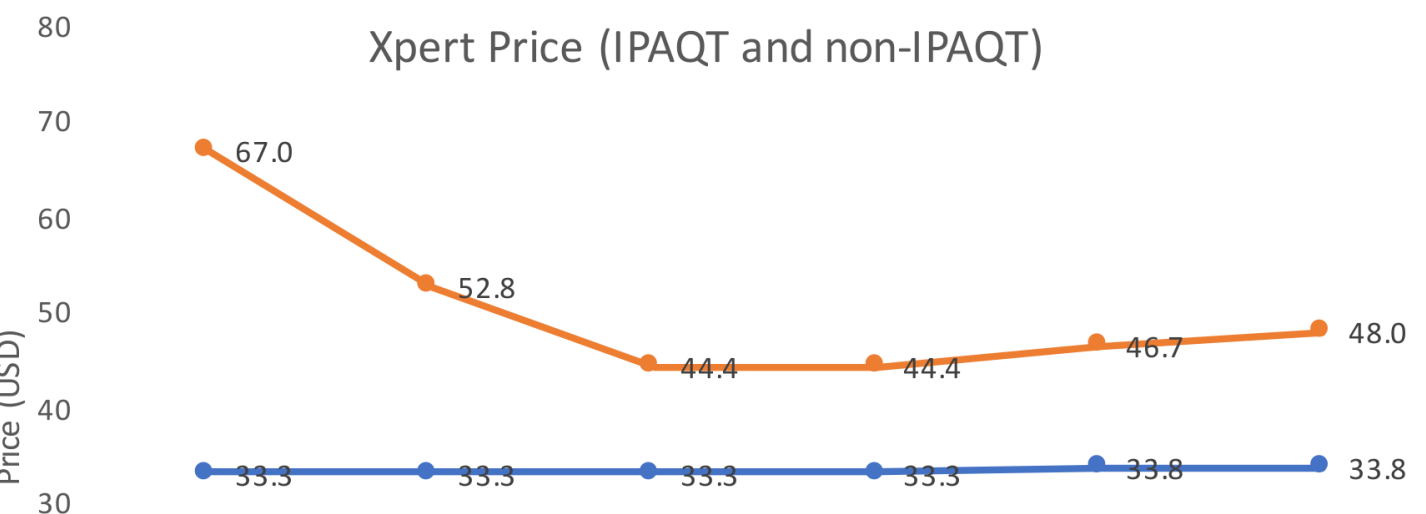

implemented in the private sector and patients paid for the tests out-of-pocket. As a result, IPAQT had to make substantial investment in aggregating private laboratories, as well as demand generation activities to complement the supply-side efforts.

Despite achieving a 10-fold increase in volumes of WHO-approved TB tests across India during a 5-year period, current testing volume under IPAQT ( $275 \mathrm{~K}$ tests) represents a small fraction of the potential demand; there are estimated 2.2 million patients with TB per year in India's private sector, ${ }^{8}$ which roughly translates to 22 million patients with presumptive TB who should be evaluated every year. Three main challenges underlie IPAQT's limited success with regard to market penetration. First, there are extensive data showing serious undertesting for $\mathrm{TB}$ in the private health sector and great reluctance of private providers to order sputumbased TB tests. ${ }^{911} 13$ Indeed, standardised patient studies in India consistently show underuse of diagnostic tests and overuse of medications, especially antibiotics. ${ }^{9-11}$ Second, IPAQT's funding allowed limited investment in demand-side activities, which tend to be highly effort intensive and resource intensive due to the fragmented base of private healthcare providers (laboratories and physicians). This was further exacerbated by the lack of technical marketing skills and resources at many smaller laboratories required to drive adoption of new technologies among uninformed providers. Third, even the reduced price of $\sim \$ 30$ for Xpert MTB/RIF, the most important new WHO-endorsed tool, is prohibitively high for a large majority of patients in India, especially those from low-income segments that comprise a large majority of India's patients with TB. These patients have higher perceived values for tangible medical interventions, such as antibiotics, steroids and intravenous fluids, that provide immediate symptomatic relief compared with diagnostic tests that only provide information. ${ }^{13}$

\section{POLICY IMPLICATIONS FOR SUSTAINABILITY}

The key to overcoming the prviously mentioned challenges and ensuring long-term sustainability of the IPAQT model depends on making the demand generation efforts more cost-effective. A few initiatives have been launched in this pursuit. From 2017, a digital marketing approach, which is less resource intensive compared with a field force model, was piloted, wherein information regarding WHO-endorsed TB tests was disseminated to private providers through a digital platform via mobile phones. It involved conventional content, such as articles and videos by experts and peers, combined with innovative interactive elements, such as gaming. Further, from early 2018, the IPAQT model was expanded to cover HCV and HIV viral load testing at lower pricing to improve the utilization of the existing capacity of testing platforms such as GeneXpert and to ensure that the marketing and capital equipment costs are apportioned over multiple tests, thereby improving financial sustainability of those activities. Until January 2019, 76 private laboratories had enrolled for HCV and HIV viral load testing under IPAQT.

Finally, IPAQT can be integrated within the broader efforts of private sector engagement that have shown the ability to change provider and patient behaviours at pilot scale in Mumbai and Patna ${ }^{18}$ and are being scaled up under the ambitious project, Joint Effort for Elimination 
of TB, launched with funding from The Global Fund. Under this project, Patient Provider Support Agencies (PPSAs) are engaging with private providers to improve the quality of TB diagnosis and treatment in 45 cities across 23 states. In addition to facilitating free testing of patients with presumptive TB in public sector laboratories, PPSAs will also disseminate information on IPAQT laboratories to willing patients who prefer and can afford paid tests in the private sector. Encouraged by these results, the IPAQT model is currently in the process of being piloted in other countries in South Asia (eg, Pakistan) and Africa, which have private markets similar to that of India.

\section{LIMITATIONS}

Our analysis capturing the impact of IPAQT has limitations owing to the design of the intervention and the associated data sources. First, IPAQT is a service-delivery project, not a research study. It was not designed as a randomised intervention. As a result, it was not possible interpret the difference in change in testing volumes between IPAQT and non-IPAQT laboratories as a causal effect of the intervention. In other words, laboratories that agreed to join the intervention were probably systematically different (eg, larger, accredited) from those who did not join. Although we conducted a survey of IPAQT laboratories to elicit their reasons for joining the intervention, the response rate was very low, which limits the generalisability and representativeness of the findings.

More fundamentally, IPAQT is a market-level (and not a laboratory-level) intervention. Hence, a rigorous evaluation would have required randomising different markets for TB diagnostic tests into control and intervention arms. Given the presence of large national laboratories, this would have meant launching the initiative in multiple countries, which was beyond the scale and scope of available resources.

As a corollary to the design of the intervention, we used data obtained from the intervention partners, that is, manufacturers and laboratories, to assess its impact. Consequently, we had to exclude some tests and laboratories from our assessment, which were either not part of the intervention or were unwilling to share their proprietary data for analysis and dissemination.

Finally, our insights regarding the effectiveness of IPAQT models are naturally restricted to diagnostic tests that require laboratory testing. An increasing number of point-of-care (POC) tests are being developed and commercialised (eg, lateral flow urine lipoarabinomannan (LAM) test), which can be conducted at the healthcare facility itself. The economics (eg, margins across different value chain entities) for these POC tests is likely to be very different compared with tests that require centralised laboratory processing. As a result, any market-making intervention may need to be designed differently.

\section{CONCLUSION}

Our results indicate the feasibility and effectiveness of a novel, market-based intervention for improving access to essential health commodities in private sector in low-income and middle-income countries, where outof-pocket spending by patients is a dominant source of healthcare financing. Such intervention is able to move private, for-profit entities from a low-volume, high-price equilibrium to a high-volume, low-price equilibrium through simultaneous demand-side (marketing and demand generation) and supply-side (modification of margins of various supply chain entities) activities. It is also feasible to expand TB-specific market interventions to include other related diseases, and this is significant within the context of universal health coverage (UHC). Long-term sustainability of such intervention will depend on the ability to integrate it with broader private sector engagement programmes and UHC investments and programmes from national and state governments.

Twitter Madhukar Pai @paimadhu

Contributors HD, PS, MP and PD oversaw the development and the implementation of the intervention. MS, APal, SS, LN, KC, PM, APar, RS, MC, $\mathrm{RK}, \mathrm{MK}, \mathrm{CO}$ and NP participated in the development and management of the intervention, including collection of data. SK, PS, PD and MP provided critical inputs on the development and intervention of the intervention and data analysis. SD provided critical inputs on the development and intervention of the intervention and data analysis, analysed the data, wrote the manuscript and made critical reviews and edits. All authors critically reviewed the manuscript. All authors agreed on the results and findings, attested to their correctness and agreed to submit the manuscript.

Funding SD's research was funded by the Bill and Melinda Gates Foundation and the Clinton Health Access Initiative. MP's research is supported by the Bill and Melinda Gates Foundation and he was a recipient of a Canada Research Chair award by the Canadian Institutes of Health Research.

Competing interests MP has previously served as a consultant to the Bill and Melinda Gates Foundation. He was involved in creating Initiative for Promoting Affordable and Quality Tuberculosis Tests (IPAQT) and also serves on the governing council of IPAQT. PD and PS were previously employed at the Bill and Melinda Gates Foundation and were involved in creating and funding IPAQT. SD has served as a consultant to the Bill and Melinda Gates Foundation. KC, PM, AP, RS, RK and MK were previously employed at the Clinton Health Access Initiative and were involved in the implementation of IPAQT.

Patient consent for publication Not required.

Provenance and peer review Not commissioned; externally peer reviewed.

Data availability statement Proprietary data regarding sales and pricing were used for analysis. These data cannot be made available publicly.

Open access This is an open access article distributed in accordance with the Creative Commons Attribution 4.0 Unported (CC BY 4.0) license, which permits others to copy, redistribute, remix, transform and build upon this work for any purpose, provided the original work is properly cited, a link to the licence is given, and indication of whether changes were made. See: https://creativecommons.org/ licenses/by/4.0/.

\section{ORCID iDs}

Sarang Deo http://orcid.org/0000-0002-3233-6014

Madhukar Pai http://orcid.org/0000-0003-3667-4536

\section{REFERENCES}

1 Wells WA, Uplekar M, Pai M. Achieving systemic and scalable private sector engagement in tuberculosis care and prevention in Asia. PLoS Med 2015;12:e1001842. 
2 Sreeramareddy CT, Qin ZZ, Satyanarayana S, et al. Delays in diagnosis and treatment of pulmonary tuberculosis in India: a systematic review. Int J Tuberc Lung Dis 2014;18:255-66.

3 Mistry N, Rangan S, Dholakia Y, et al. Durations and delays in care seeking, diagnosis and treatment initiation in uncomplicated pulmonary tuberculosis patients in Mumbai, India. PLoS One 2016;11:e0152287.

4 Mistry N, Lobo E, Shah S, et al. Pulmonary tuberculosis in Patna, India: durations, delays, and health care seeking behaviour among patients identified through household surveys. J Epidemiol Glob Health 2017;7:241-8.

5 Cazabon D, Alsdurf H, Satyanarayana S, et al. Quality of tuberculosis care in high burden countries: the urgent need to address gaps in the care cascade. Int $J$ Infect Dis 2017;56:111-6.

6 Pai M, Furin J. Tuberculosis innovations mean little if they cannot save lives. Elife 2017;6:e25956.

7 Ponnudurai N, Denkinger CM, Van Gemert W, et al. New TB tools need to be affordable in the private sector: the case study of Xpert MTB/RIF. J Epidemiol Glob Health 2018;8:103-5.

8 Arinaminpathy $\mathrm{N}$, Batra $\mathrm{D}$, Khaparde $\mathrm{S}$, et al. The number of privately treated tuberculosis cases in India: an estimation from drug sales data. Lancet Infect Dis 2016;16:1255-60.

9 Das J, Kwan A, Daniels B, et al. Use of standardised patients to assess quality of tuberculosis care: a pilot, cross-sectional study. Lancet Infect Dis 2015;15:1305-13.

10 Satyanarayana S, Kwan A, Daniels B, et al. Use of standardised patients to assess antibiotic dispensing for tuberculosis by pharmacies in urban India: a cross-sectional study. Lancet Infect Dis 2016;16:1261-8.

11 Kwan A, Daniels B, Saria V, et al. Variations in the quality of tuberculosis care in urban India: a cross-sectional, standardized patient study in two cities. PLoS Med 2018;15:e1002653.

12 Jarosławski S, Pai M. Why are inaccurate tuberculosis serological tests widely used in the Indian private healthcare sector? A rootcause analysis. J Epidemiol Glob Health 2012;2:39-50.

13 McDowell A, Pai M. Treatment as diagnosis and diagnosis as treatment: empirical management of presumptive tuberculosis in India. Int J Tuberc Lung Dis 2016;20:536-43.

14 Grenier J, Pinto L, Nair D, et al. Widespread use of serological tests for tuberculosis: data from 22 high-burden countries. Eur Respir J 2012;39:502-5.

15 Steingart KR, Flores LL, Dendukuri N, et al. Commercial serological tests for the diagnosis of active pulmonary and extrapulmonary tuberculosis: an updated systematic review and meta-analysis. PLoS Med 2011;8:e1001062.

16 World Health Organization. Commercial serodiagnostic tests for diagnosis of tuberculosis: policy statement. Geneva: World Health Organization, 2011.

17 WHO. Standards of TB care in India, 2014. Available: http://www. searo.who.int/india/mediacentre/events/2014/stci book.pdf

18 Division CT, Office WIC. Universal access to TB care (UATBC): concurrent assessment report, 2016. Available: http://www.searo. who.int/india/publications/universal_access_to_tb_care_part1.pdf 